\title{
The Minimum Percentage of Triolein Emulsion for Studying Cerebral Vascular Permeability with Least Brain Edema
}

\author{
Seon Hee Choi ${ }^{1,2}$; Hak Jin Kim ${ }^{1,3,{ }^{*}}$; Lee Hwangbo ${ }^{1,2}$; Yong-Woo Kim ${ }^{1,4}$ \\ ${ }^{1}$ Department of Radiology, Pusan National University School of Medicine, Yangsan, Republic of Korea \\ ${ }_{3}^{2}$ Pusan National University Postgraduate School, Yangsan, Republic of Korea \\ 3 Biomedical Research Institute, Pusan National University Hospital, Republic of Kore \\ ${ }^{4}$ Biomedical Research Institute, Pusan National University Hospital, Republional University, Yangsan Hospital, Yangsan, Republic of Korea \\ *Corresponding author: Hak Jin Kim, Department of Radiology, Pusan National University School of Medicine, Biomedial Research Institute, Pusan National University Hospital, 10 \\ 1-Ga, Ami-Dong, Seo-Ku, Pusan, 602-739, Republic of Korea. Tel: +82-512407371, Fax: +82-512447534, E-mail: hakjink@pusan.ac.kr
}

Received: September 16, 2013; Revised: November 13, 2013; Accepted: May 2, 2014

\begin{abstract}
Background: Triolein emulsion infusion into the brain produces transiently increased vascular permeability.
Objectives: The purpose of this study was to find the minimum percentage of triolein emulsion required for studying vascular permeability with minimal brain edema.

Materials and Methods: Sixty healthy cats were divided into six groups according to the concentration of emulsified triolein infused into the carotid artery: group 1, 0.125\% $(n=10)$; group 2, 0.25\% $(n=10)$; group 3, $0.5 \%(n=10)$; group 4, $1 \%(n=10)$; group 5, $2 \%(n=10)$; and group 6, saline infusion (control group, $\mathrm{n}=10$ ). T2-, T1- and contrast enhanced T1-weighted MR images were obtained 2 hours after infusing triolein emulsion. Contrast enhancement ratios (CERs) and signal intensity ratios (SIRs) versus contralateral hemispheres were calculated. Statistical analysis was performed by analysis of variance followed by Tukey's test. P values of $\leq 0.05$ were considered significant.

Results: The lesion hemispheres showed mild hyperintensity due to edema on T2-weighted images, and contrast enhancement on postcontrast T1-weighted images in cats of group 1-5. CERs showed statistically significant differences between the control group and group 3 $(\mathrm{P}=0.006)$, group $4(\mathrm{P}=0.003)$, and group $5(\mathrm{P}<0.001)$. However, SIRs were significantly different between the control group and group 5 only $(\mathrm{P}<0.001)$.

Conclusion: The minimum concentration of triolein emulsion required to increase vascular permeability adequately with minimal brain edema in a cat model was $0.5 \%$.
\end{abstract}

Keywords: Triolein; Emulsions; Blood-Brain Barrier; Vascular Permeability; MRI

\section{Background}

The blood brain barrier (BBB) forms a structural and functional barrier between the blood circulation and brain parenchyma, regulates the transport of molecules, and prevents blood cells accessing brain tissues. However, the BBB acts as a barrier that prevents drug delivery to the brain. Since the transport of most therapeutic agents is restricted by the BBB, new methods have been sought to overcome this protective function of the $\mathrm{BBB}$, because enhanced drug transport across the $\mathrm{BBB}$ could facilitate the treatment of brain tumors, cancer metastases to the brain, and other brain diseases (1-3). The efficient delivery of diagnostic agents across the BBB would also be beneficial for improving the detection of pathologic targets inside the brain. Certain pathological conditions, such as stroke, traumatic brain injury, and inflammation disrupt the BBB (4), but these disruptions are inappropriate for clinical applications, since BBB destruction associated with disease can lead to brain damage.

In a previous study, triolein emulsion transiently increased vascular permeability with interstitial edema in the cat brain when administrated via the carotid artery, as determined by MR imaging and microscopic examination (5). Increased vascular permeability of the BBB could be applicable to experiments on drug reactions in the brain or in studies on the treatment of CNS diseases. However, excessive cerebral edema has been reported in many triolein emulsion studies, and this could have unpredictable side effects. Thus, the dosage of triolein emulsion required to increase vascular permeability while minimizing interstitial edema should be optimized. In previous studies, the amount of triolein emulsion (0.05$0.2 \mathrm{~mL}$ triolein in $20 \mathrm{~mL}$ saline) administered have varied widely (6-8), and dosages were not optimized. Kim et al. (9) reported that the proper dosage of triolein emulsion required to increase vascular permeability with minimal edema appears to be approximately $3 \mathrm{~mL} / \mathrm{kg}$ of triolein in a cat model. However, this study was limited to $0.5 \%$ triolein emulsion. In fact, no report has been issued on the relationship between vascular permeability and interstitial edema using triolein emulsions of different concentrations.

Copyright ( ) 2014, Tehran University of Medical Sciences and Iranian Society of Radiology; Published by Kowsar. This is an open-access article distributed under the terms of the Creative Commons Attribution-NonCommercial 4.0 International License (http://creativecommons.org/licenses/by-nc/4.0/) which permits copy and redistribute the material just in noncommercial usages, provided the original work is properly cited. 


\section{Objectives}

The purpose of this study was to establish the minimum triolein emulsion concentration suitable for studying vascular permeability with minimal brain edema in a cat model.

\section{Materials and Methods}

\subsection{Experimental Preparations}

All animal experiments were conducted in accordance with the guidelines issued by our Institutional Animal Care and Use Committee (Approval No: 2012-038). Sixty healthy adult domestic male cats weighing 2.8-3.3 kg were anesthetized with an intramuscular injection of ketamine $\mathrm{HCl}$ (2.5 mg/kg; Huons, Jechun, Korea) and xylazine (0.125 mg/kg; Bayer Korea, Seoul, Korea). Cats were allowed to breathe ambient air spontaneously during the procedure. Briefly, the right femoral artery was isolated and its distal portion was ligated with 4.0 silk. An 18-guage intravenous catheter was then inserted into the artery proximal to the ligated portion. A 2.2F microcatheter (Progreat microcatheter, Terumo, Tokyo, Japan) equipped with a microguidewire (GT guidewire, Terumo, Tokyo, Japan) was then passed through the intravenous catheter into the lumen of the artery. The tip of the microcatheter was positioned in the right or left common carotid artery at the level of the fourth cervical vertebra under fluoroscopic guidance. Triolein (triglyceride, 1, 2, 3-tri [cis-9-octadecenoyl] glycerol) was supplied by SigmaAldrich (St. Louis, MO, USA).

\subsection{Triolein Emulsion Infusion}

A $1 \mathrm{~mL}$ syringe containing triolein and another $20 \mathrm{~mL}$ syringe containing saline were connected to a three-way stopcock. Triolein emulsion was prepared by mixing triolein and saline via the stopcock using a vigorous to-andfro movement of the syringes for $2 \mathrm{~min}$. Sixty cats were divided into six groups ( $\mathrm{n}=10$ in each group) according to the concentration of the triolein emulsion infused: group 1, $0.125 \%$ (0.025 mL triolein in $20 \mathrm{~mL}$ of saline); group 2, 0.25\% (0.05 mL in $20 \mathrm{~mL}$ ); group 3, 0.5\% (0.1 mL in $20 \mathrm{~mL}$ ); group $4,1 \%$ triolein emulsion $(0.2 \mathrm{~mL}$ in 20 $\mathrm{mL}$ ); group 5, $2 \%$ (0.4 mL in $20 \mathrm{~mL})$; and group 6 , saline only (the control group) (Table 1). Triolein emulsion was infused at $3 \mathrm{~mL} / \mathrm{Kg}$ of body weight at a rate of $4 \mathrm{~mL} / \mathrm{min}$ for 5 minutes, as described by Kim et al. (9). After administering triolein, the microcatheter was removed from the femoral artery and the artery was ligated.

\subsection{MR imaging}

MRI was performed 2 hours after triolein infusion. Each cat was placed in the prone position within a pediatric MR infant restrainer, and a flexible radiofrequency coil (Siemens, Erlangen, Germany) was placed around the head. All studies were performed using a 3T MR Tim Trio scanner (Siemens, Erlangen, Germany). Images were acquired in the coronal plane. For spin-echo imaging, the following scan parameters were employed: for T2weighted images (T2WIs), repetition time $[\mathrm{TR}]=3000$ $\mathrm{ms}$, echo time $[\mathrm{TE}]=96 \mathrm{~ms}$ and bandwidth $=78 \mathrm{~Hz} /$ pixel, and for T1-weighted images (T1WIs), TR/TE $=320 / 20 \mathrm{~ms}$ and bandwidth $=65 \mathrm{~Hz} /$ pixel. In both imaging modes, the following were used; section thickness $=4 \mathrm{~mm}$ with a $0.1 \mathrm{~mm}$ gap, field of view $=70-75 \mathrm{~mm}$, two excitations, and acquisition matrix $=210 \times 256$. For contrast studies, $0.3 \mathrm{~mL} / \mathrm{kg}$ meglumine (Megaray Dongkuk Pharma, Seoul) was injected intravenously. T2W, T1W, and post-contrast T1W images were analyzed. Signal intensities (SI) were measured quantitatively in ipsilateral and contralateral hemispheres on T1W, T2W, and post-contrast T1W images. Seven slices were obtained per scan. SIs and areas of hemispheres were measured blindly using a manually drawn region of interest (ROI) in the whole brain on the second console of the MR machine. Manually drawn ROI on T1W images were automatically set on T2W and postcontrast T1W images on the second console (Syngo Siemens, Erlangen, Germany). Thus, the shapes, positions, and sizes of the ROIs were identical on T1W, T2W, and postcontrast T1W images. Entire hemispheres were included in measurements, but large superficial cerebral vessels were excluded. First and last images were excluded from SI measurements because of overlapping artifacts. The SIs of five consecutive slices were tabulated and the mean contrast enhancement ratios (CERs) of ipsilateral/contralateral hemisphere (CERs $=$ [SI on post-contrast T1WIs-SI on pre-contrast TiWIs]/SI on pre-contrast TiWIs) were calculated. Mean signal intensity ratios (SIRs) in five consecutive slices were calculated by dividing the T2WI SIs of ipsilateral hemispheres by those of contralateral hemispheres. SIRs and CERs were compared between groups.

\subsection{Statistical Analysis}

Data were statistically evaluated by analysis of variance, followed by Tukey's honestly significant difference test to compare differences between the mean CERs of ipsilateral and contralateral hemispheres and mean

Table 1. Means and Standard Deviations (SD) of Body Weights and the Concentrations of Triolein Emulsion Infusion in the Six Study Groups

\begin{tabular}{lccc}
\hline Group & No. & $\begin{array}{c}\text { Body Weight } \\
(\mathbf{k g})^{\mathrm{a}}\end{array}$ & $\begin{array}{c}\text { Triolein Emulsion (TE) or } \\
\text { Saline Infusion }\end{array}$ \\
\hline $\mathbf{1}$ & 10 & $3.0 \pm 0.8$ & TE $0.125 \%$ \\
$\mathbf{2}$ & 10 & $2.8 \pm 0.2$ & TE 0.25\% \\
$\mathbf{3}$ & 10 & $3.5 \pm 0.6$ & TE 0.5\% \\
$\mathbf{4}$ & 10 & $2.7 \pm 0.7$ & TE $1 \%$ \\
$\mathbf{5}$ & 10 & $2.9 \pm 0.8$ & TE $2 \%$ \\
$\mathbf{6}$ & 10 & $3.3 \pm 0.4$ & saline \\
\hline a & &
\end{tabular}


Hee Choi S et al.

SIRs on T2W images. Calculated two-tailed P values of $\leq$ 0.05 were considered statistically significant. CER and T2 SIR measurements of each group were tested for normality by using Kolmogorov-Smirnov/Lilliefor test, and the normality was not rejected $(\mathrm{P}>0.05)$. Additionally, Pearson's correlation coefficient was calculated to assess the correlation between CER and T2 SIR. Simple linear regression analysis was also performed on these two variables. Both $\mathrm{R}$ (version 2.15.3, R foundation for statistical computing, Vienna, Austria) and SPSS (version 18.0, Chicago, Ill, USA) software were used for analysis of the data and constructing the plots.

\section{Results}

Ipsilateral hemispheres showed mild hyperintensity on T2-weighted images, and contrast enhancement on postcontrast T1-weighted images. Group 5 tended to show more $\mathrm{T} 2$ hyperintensity and more contrast enhancement (Figure 1) than the other groups. These observations were usually weak in groups 1 and 2 . There were contralateral paramedian lesions in two cats in group 3, three cats in group 4, and four cats in group 5. CER differences were statistically significant between the control group (group 6) and groups $3(\mathrm{P}=0.006), 4(\mathrm{P}=0.003)$, and 5 ( $\mathrm{P}$ $<0.001$ ) (Figure 2). However, no statistical difference was observed between groups 3,4 , and $5(\mathrm{P}=0.9983$ between groups 3 and $4, \mathrm{P}=0.3042$ between groups 3 and $5, \mathrm{P}=$ 0.1109 between groups 4 and 5 ).

T2 SIRs were significantly different between the control group and group 5 only $(\mathrm{P}<0.001)$ (Figure 3$)$. No statistical difference was observed between group $1(\mathrm{P}=0.5192)$, group $2(\mathrm{P}=0.2099)$, group $3(\mathrm{P}=0.1234)$, group $4(\mathrm{P}=$ 0.1711 ) and the control group (group 6).

Simple linear regression analysis of CER and T2 SIR were done on 47 test group cats after excluding three outliers. The three outliers were identified by using the residualversus-fitted plot acquired from linear regression from all 50 test subjects. There was a positive correlation between the two variables, and about $32.06 \%$ of variation in CER ratio was explained by T2 SIR, or vice versa (Figure 4, R-squared $=0.3206$, p-value $<0.001)$. In other words, increased brain permeability in the test subjects explained about one-third variability in brain edema on MR.
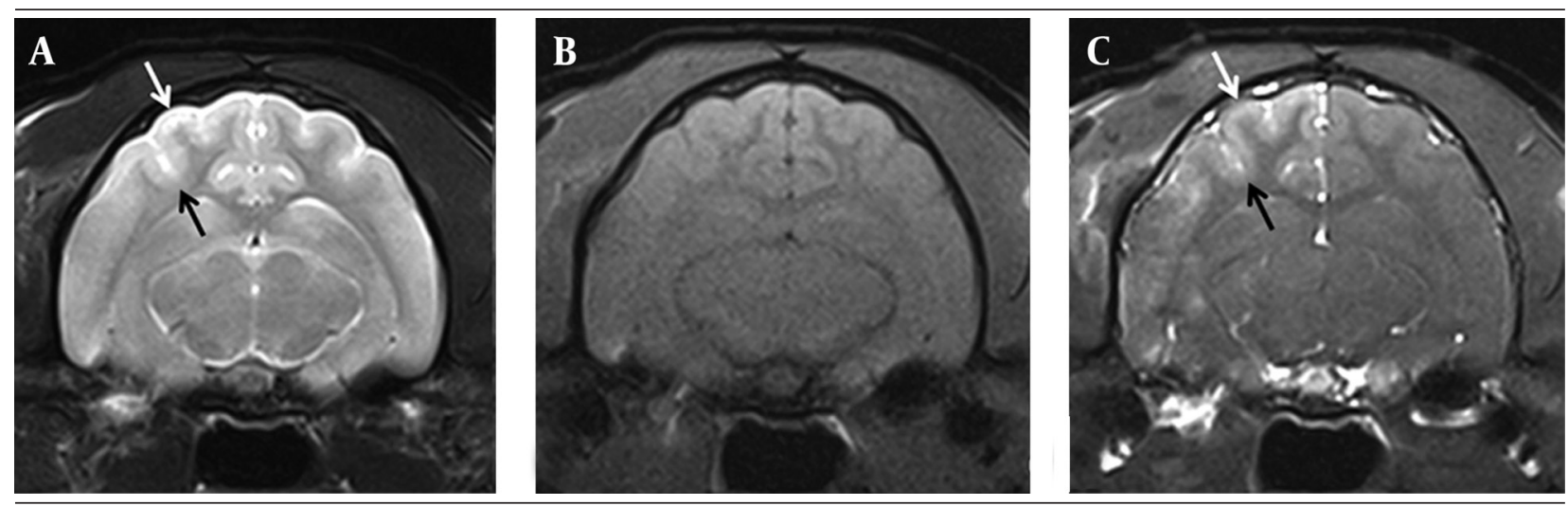

Figure 1. MR images of a cat in group 5 infused with $2 \%$ triolein emulsion ( $3 \mathrm{ml} / \mathrm{kg}$ of body weight) into the right carotid artery. T2-weighted image (A) shows mild hyperintensity on the superficial cortex of the ipsilateral hemisphere (arrows). (B) The lesion was isointense on T1-weighted images and (C) shows diffuse enhancement on Gd-enhanced T1-weighted images (arrows).

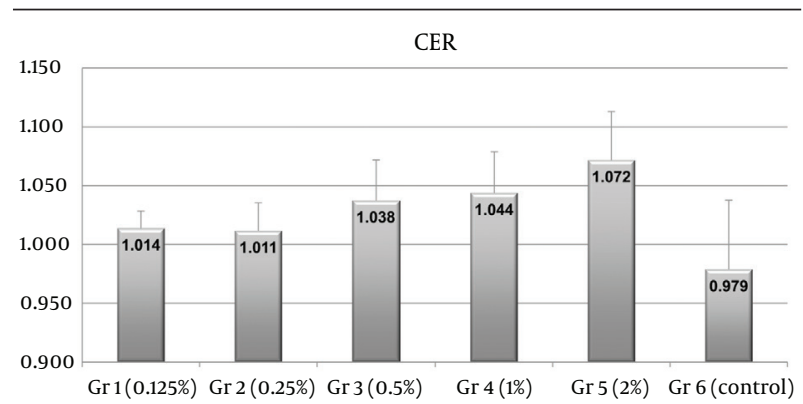

Figure 2. Plot of contrast enhancement ratios (CERs) on Gd-T1-weighted images. CERs of groups 3, 4, and 5 were significantly higher than those of saline treated controls (group 6) $(\mathrm{P}=0.006,0.003$ and $<0.001$, respectively). CERs of groups 1 and 2 were slightly higher than those of controls $(P=0.301$ and 0.407 , respectively). No significant difference was found between the CERs of groups 3, 4 and $5(\mathrm{P}=0.9983$ between group 3 and $4, \mathrm{P}=0.3042$ between group 3 and $5, \mathrm{P}=0.1109$ between group 4 and 5 ). Numbers on the tops of the bars indicate mean group CERs.

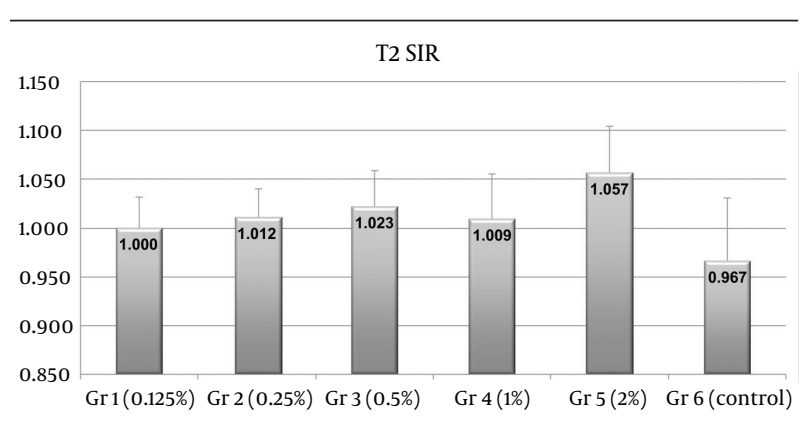

Figure 3. Plot of signal intensity ratios (SIRs) on T2-weighted images. SIRs in group 5 were significantly higher than those of saline treated controls (group 6) $(\mathrm{P}<0.001)$. Numbers on the tops of bars indicate mean group SIRs. 
Hee Choi S et al.

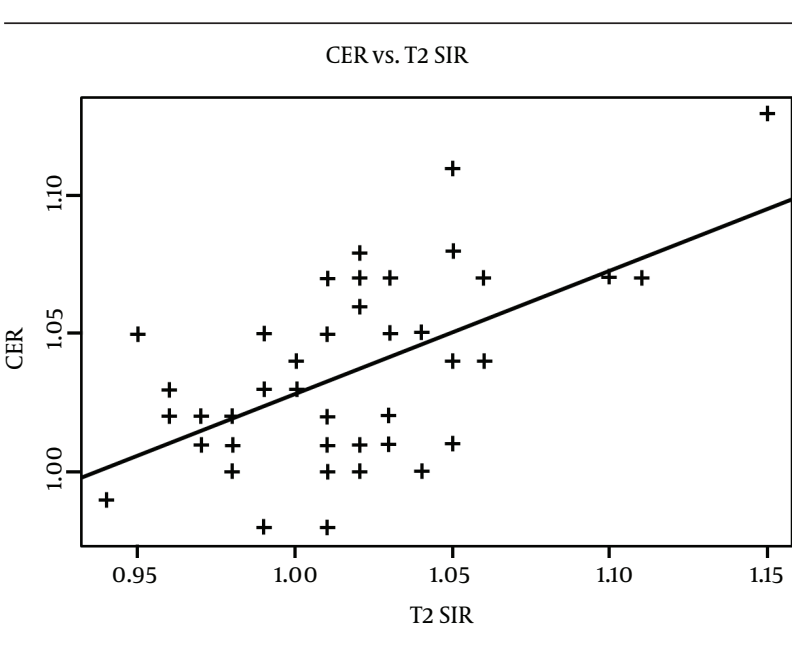

Figure 4. Scatter plot of CER ratio and T2 SIR values. The y-axis represents contrast enhancement between ipsilateral and contralateral hemispheres on post-contrast T1-weighted images. The $\mathrm{x}$-axis represents signa intensity ratios between ipsilateral and contralateral hemispheres on T2 weighted images. The regression line shows a significant positive correlation between CER and T2 SIR values (R-squared $=0.3206$, P value $<0.001$ ).

\section{Discussion}

The development of a strategy for controlled, transient BBB disruption would be beneficial for basic research and clinical applications for many brain diseases, especially intractable brain tumors. Triolein emulsion has been reported to increase vascular permeability transiently when it is infused into the brain via a carotid artery. This increased vascular permeability may be observed as homogenous contrast enhancement on Gd-T1-weighted images and hyperintensity on T2-weighted images due to BBB breakdown by triolein as has been reported in the previous studies $(5,10)$. However, undesirable vasogenic edema could cause unpredictable side effects and adversely affect laboratory experiments or studies on the clinical effects of treatments. Thus, an optimized triolein emulsion dosage is required that increases vascular permeability without causing undue interstitial edema.

In the present study, contrast enhancement was statistically significant when triolein emulsion was infused via a carotid artery into cats at concentrations above $0.5 \%$ ( $0.1 \mathrm{~mL}$ of triolein in $20 \mathrm{~mL}$ of saline), and increased in a dose-dependently manner. Regarding vasogenic edema, T2-weighted images revealed no significant parenchymal edema when triolein emulsion was infused at $1 \%$. Thus, the major finding of this study is that the minimum concentration of triolein emulsion required to increase vascular permeability adequately without brain edema is $0.5 \%$. The lesion conspicuity was more prominent on Gd-T1-weighted image than T2-weighted image in the present study. In previous studies $(5,7,9)$, triolein emulsion has been used at $0.25-1 \%$, but without knowledge of the optimal triolein emulsion concentration. The present study describes the relation between triolein emulsion concentration, vascular permeability, and brain edema.
In fact, vascular permeability and vasogenic edema were found to be linearly correlated.

Another important consideration in studies of vascular permeability change is the total volume of triolein infused. Ryu et al. (9) suggested that the minimum dosage of triolein emulsion ( $0.5 \%$ ) required to achieve increased vascular permeability in the cat brain appears to be $3 \mathrm{~mL} /$ $\mathrm{kg}$. However, the authors used only one concentration of triolein emulsion. Thus, according to the study by Ryu et al. (9) and the present study, the optimum dosage and concentration of triolein emulsion to achieve increased vascular permeability with minimal edema in a cat model appears to be $3 \mathrm{~mL} / \mathrm{kg}$ at a triolein emulsion concentration of $0.5 \%$.

In the present study, Gd-T1-weighted images were used to detect BBB permeability changes. Contrast enhanced T1-weighted images are able to show sites of BBB breakdown (11). In the presence of increased permeability or disruption of the BBB, gadolinium extravasates and diffuses into the brain parenchyma. In the present study, significant differences in contrast enhancement were observed between the control group and groups infused with triolein emulsion at more than $0.5 \%$. Contrast enhancement indicating increased vascular permeability by triolein emulsion infusion into the brain is well known in experimental animal studies $(5,7,9)$. In a study of BBB disruption induced by emulsified oleic acid, nitric oxide was found to contribute to oleic acid-induced activations of matrix metalloproteinase- 2 and -13 and the development of transient brain edema. After exposure to oleic acid administered intra-arterially, endothelial cells are stimulated and intercellular adhesion molecule-1 expression on the luminal surface occurs. Intercellular adhesion molecule- 1 then recruits neutrophils from the blood circulation to affected brain vessels. These neutrophils stay close to vessels or infiltrate brain parenchyma and express inducible nitric oxide synthase, which produces nitric oxide and triggers the activations of matrix metalloproteinase- 2 and -13 in the endothelial cells. Activated matrix metalloproteinase- 2 and -13 then degrade laminin and the basal lamina structure, and ultimately, the BBB is disrupted and brain edema develops (12). However, the mechanism of BBB disruption by triolein emulsion has not been elucidated.

Observed T2 hyperintensity has been attributed to vasogenic edema induced by BBB breakdown in the previous triolein studies $(5,10)$. In the present study, a significant difference was observed between $\mathrm{T} 2$ hyperintensities in the control group and the $2 \%$ triolein group. In other words, no significant difference was observed in brain edema when the triolein emulsion concentration was less than $2 \%$. A previous study of $0.5 \%$ triolein emulsion infusion into the cat brain showed that $\mathrm{T} 2$ hyperintensity occurred 1 hour after infusion, and that this hyperintensity diminished rapidly and approached normal 4 days after infusion (5). In the present study, T2-weighted MR imaging showed that brain edema is not excessive when 
$0.5 \%$ triolein emulsion is used. However, the correlation between the amount of triolein infused and the clinical effects of increased vascular permeability or vasogenic edema have not been studied yet in an experimental animal. Furthermore, the optimal dosage may depend on the purpose of the study.

In the present study, there were contralateral paramedian lesions in two cats in group 3, three cats in group 4, and four cats in group 5. These contralateral lesions were probably due to the communication of two hemispheres similar to the Willis circle in humans. Velocity of infusion and the total amount of triolein emulsion might be related to those contralateral lesions.

Temporary BBB disruption could be useful in increasing drug delivery to tumors and adjacent brain parenchyma, and BBB disruption using hyperosmolar mannitol has been used for this purpose (13), though its efficacy is controversial (14). Drug delivery is most effective when a drug is administered within 5-10 min of hyperosmolar BBB disruption by mannitol (14), but this time window appears to be inadequate for drug delivery to brain tissues. On the other hand, the time required for barrier opening is much longer when triolein is used $(5,10)$. Opening of the BBB may have a substantial clinical impact on the treatment of patients with intractable brain tumors, and an adequate increase in vascular permeability by triolein emulsion infusion could make it possible to increase therapeutic response or lower the amounts of chemotherapeutic agents administered (9).

Infarction as a side effect can also happen after triolein emulsion infusion into the brain. The size of the triolein particle in the emulsion of the present study was not uniform. This was because there had been no ideal method to make the particle uniform and to choose the particle size. Thus, if a large-sized particle of triolein enters the carotid artery, there could be occlusion of the vessel and it may result in brain infarction. Hemorrhage is another side effect after infusion of triolein (especially bolus triolein) due to mechanical disruption of the endothelial wall by triolein (10). However, two hours after triolein emulsion infusion used in the present study is not sufficient to assess these side effects; longer follow-up time and further studies are necessary.

This study had several limitations. In the present study, the minimum concentration of triolein emulsion that significantly increased vascular permeability, but not edema was determined by MRI. Furthermore, we did not examine clinical symptoms or signs during the study, and their use could have resulted in different findings. Accordingly, we suggest that in future experiments, relations between triolein emulsion infusion concentrations and clinical outcomes should be studied.

The present study shows that the minimum concentration of triolein emulsion required to significantly increase vascular permeability with minimal edema in the cat brain at a dose of $3 \mathrm{~mL} / \mathrm{Kg}$ appears to be $0.5 \%$. T2weighted and contrast-enhanced MR images revealed that vascular permeability and brain edema were increased by infusing triolein emulsion into a carotid artery. We believe the minimum concentration of triolein emulsion required to produce vascular permeability and brain edema when infused into the cat carotid artery as determined by the present study provides useful basic data for future studies on CNS drug delivery models.

\section{Authors' Contributions}

Choi SH and Kim YW contributed to data collection, preparing the manuscript, and analysis. Kim HJ contributed to study concept, design, quality assessment of the included papers, preparing the manuscript, and supervision. Hwangbo L contributed to the statistical analysis.

\section{References}

1. Neuwelt EA. Mechanisms of disease: the blood-brain barrier. Neurosurgery. 2004;54(1):131-40.

2. Drappatz J, Wen PY. Chemotherapy and targeted molecular therapies for brain metastases. Expert Rev Neurother. 2006;6(10):146579.

3. Cavaliere R, Schiff D. Chemotherapy and cerebral metastases: misperception or reality? Neurosurg Focus. 2007;22(3):E6.

4. Abbott NJ, Ronnback L, Hansson E. Astrocyte-endothelial interactions at the blood-brain barrier. Nat Rev Neurosci. 2006;7(1):41-53.

5. Kim HJ, Lee CH, Kim HG, Lee SD, Son SM, Kim YW, et al. Reversible MR changes in the cat brain after cerebral fat embolism induced by triolein emulsion. AJNR Am J Neuroradiol. 2004;25(6):958-63.

6. Kim KN, Kim HJ, Lee SD, Moon TY, Lee SH, Lee JW, et al. Effect of triolein emulsion on the blood-testis barrier in cats. Invest Radiol. 2004;39(7):445-9.

7. Kim YW, Kim HJ, Cho BM, Moon TY, Eun CK. The study of cerebral hemodynamics in the hyperacute stage of fat embolism induced by triolein emulsion. AJNR Am J Neuroradiol. 2006;27(2):398-401.

8. Baik SK, Kim YW, Kim HJ, Lee JW, Cho BM, Kim DH, et al. Proton magnetic resonance spectroscopic findings of cerebral fat embolism induced by triolein emulsion in cats. Acta Radiol. 2008;49(10):1174-81.

9. Ryu JH, Jeong YJ, Kim HJ, Kim DH, Kim DC, Kim MJ, et al. MRI of the cat brain in the study of vascular permeability after intraarterial carotid injection of triolein. Acta Radiol. 2010;51(5):563-8.

10. Kim HJ, Lee CH, Lee SH, Cho BM, Kim HK, Park BR, et al. Early development of vasogenic edema in experimental cerebral fat embolism in cats: correlation with MRI and electron microscopic findings. Invest Radiol. 2001;36(8):460-9.

11. Barzo P, Marmarou A, Fatouros P, Corwin F, Dunbar J. Magnetic resonance imaging-monitored acute blood-brain barrier changes in experimental traumatic brain injury. J Neurosurg. 1996;85(6):1113-21.

12. Han HS, Jang JH, Park JS, Kim HJ, Kim JK. Transient blood brain barrier disruption induced by oleic acid is mediated by nitric oxide. Curr Neurovasc Res. 2013;10(4):287-96.

13. Gumerlock MK, Belshe BD, Madsen R, Watts C. Osmotic bloodbrain barrier disruption and chemotherapy in the treatment of high grade malignant glioma: patient series and literature review. J Neurooncol.1992;12(1):33-46.

14. Zunkeler B, Carson RE, Olson J, Blasberg RG, DeVroom H, Lutz RJ, et al. Quantification and pharmacokinetics of blood-brain barrier disruption in humans. J Neurosurg. 1996;85(6):1056-65. 